\title{
Discovery of necrosis avidity of rhein and its applications in necrosis imaging
}

\section{Dongjian Zhang, Qiaomei Jin, Yicheng Ni \& Jian Zhang}

To cite this article: Dongjian Zhang, Qiaomei Jin, Yicheng Ni \& Jian Zhang (2020): Discovery of necrosis avidity of rhein and its applications in necrosis imaging, Journal of Drug Targeting, DOI: 10.1080/1061186X.2020.1759079

To link to this article: https://doi.org/10.1080/1061186X.2020.1759079

Accepted author version posted online: 21

Apr 2020.

Submit your article to this journal $\sqsubset$

山ll Article views: 3

Q View related articles $\widetilde{ }$

View Crossmark data 


\section{Discovery of necrosis avidity of rhein and its applications in necrosis}

\section{imaging}

Dongjian Zhang ${ }^{\mathrm{a}, \mathrm{b}}$, Qiaomei Jin ${ }^{\mathrm{a}, \mathrm{b}}$, Yicheng Ni ${ }^{\mathrm{c}}$, and Jian Zhang ${ }^{\mathrm{a}, \mathrm{b}}$

${ }^{a}$ Affiliated Hospital of Integrated Traditional Chinese and Western Medicine, Nanjing University of Chinese Medicine, Nanjing 210028, P.R. China

${ }^{\mathrm{b}}$ Laboratories of Translational Medicine, Jiangsu Province Academy of Traditional Chinese Medicine, Nanjing 210028, P.R. China

${ }^{\mathrm{c}}$ Theragnostic Laboratory, Campus Gasthuisberg, KU Leuven, Leuven 3000, Belgium

Dongjian Zhang and Qiaomei Jin contributed equally to this work.

Correspondence to: Dr. Jian Zhang, No.100, Shizi Street, Hongshan Road, Nanjing 210028, Jiangsu Province, P.R.China, E-mail: zjwonderful@hotmail.com, Tel: +86-25-52362107, Fax: +86-25-85637817

The ORCID for each author is as follows:

Dongjian Zhang: 0000-0003-4821-6620

Qiaomei Jin: 0000-0002-8559-9125

Yicheng Ni: 0000-0002-8553-7565

Jian Zhang: 0000-0002-8402-9753 


\begin{abstract}
Necrosis-avid agents possess exploitable theragnostic utilities including evaluation of tissue viability, monitoring of therapeutic efficacy as well as diagnosis and treatment of necrosis-related disorders.

Rhein (4,5-dihydroxyl-2-carboxylic-9,10-dihydrodiketoanthracene), a naturally occurring monomeric anthraquinone compound extensively found in medicinal herbs, was recently demonstrated to have a newly discovered necrosis-avid trait and to show promising application in necrosis imaging. In this overview, we present the discovering process of rhein as a new necrosis-avid agent as well as its potential imaging applications in visualization of myocardial necrosis and early evaluation of tumor response to therapy. Moreover, the molecular mechanism exploration of necrosis avidity behind rhein are also presented. The discovery of necrosis avidity with rhein and the development of rhein-based molecular probes may further expand the scope of necrosis-avid compounds and highlight the potential utility of necrosis-avid molecular probes in necrosis imaging.
\end{abstract}

Keywords: Rhein, necrosis avidity, molecular imaging, visualization of myocardial necrosis, monitoring of therapeutic efficacy, mechanism exploration 


\section{Introduction}

Necrosis avidity of small molecular compounds is a unique property with exploitable theragnostic potentials [1,2]. It has been more than 45 years since the first radiolabeled small molecular agent ${ }^{99 \mathrm{~m}} \mathrm{Tc}$-pyrophosphate was found to be avid for necrotic myocardium [3]. During recent 25 years, a large number of small molecular compounds of high affinity for necrosis have been identified, developed and evaluated in animal models of different diseases including acute myocardial infarction (AMI), cerebral infarction, tumor, atherosclerosis, gastric ulcer, etc. [1,2,4-13], showing potential utilities in evaluation of tissue viability, monitoring of therapeutic efficacy as well as diagnosis and treatment of necrosis-related disorders [1,2,4-8]. These small molecular compounds sharing similar affinity for necrosis have been categorized by $\mathrm{Ni}$ et al. as necrosis-avid contrast agents (NACAs) or necrosis-avid compounds/agents (NACs/NAAs) [1,2,4-6], which are also adopted by others [10-16], although they do appear diverse in terms of chemical structures, physiochemical properties, mechanisms of action, potential applications, etc. [1,2,4-6,10-13,17].

Rhein (4,5-dihydroxyl-2-carboxylic-9,10-dihydrodiketoanthracene, Figure 1), a naturally occurring monomeric anthraquinone compound extensively found in medicinal herbs such as Rheum palmatum L., Polygonum multiflorum Tunb., Aloe barbadensis Miller and Cassia tora L., has been demonstrated to exhibit extensive pharmacological activities including hepatoprotective, nephroprotective, anti-inflammatory, antioxidant, anticancer, antidiabetic, antimicrobial, purgative, lipid-lowering effects, etc.[18-20]. More recently, based on our previous studies on necrosis avidity of bisanthracene nuclear anthraquinone compounds [21-43] and in pursuit for NAAs with improved physicochemical properties, pharmacokinetics and biodistribution, monomeric anthraquinone compound rhein was demonstrated to have a newly discovered necrosis-avid trait and to show promising applications in noninvasive visualization of myocardial necrosis and early monitoring of tumor response to therapy [44-49]. 
In this mini-review, we present the discovering process of rhein as a new NAA as well as its potential applications in necrosis imaging including visualization of myocardial necrosis and early evaluation of tumor response to therapy. Moreover, the exploration on molecular mechanism of necrosis avidity of rhein are also presented. The discovery of necrosis avidity with rhein and the development of rhein-based molecular probes may further expand the scope of NAAs and highlight the potential utility of NAAs in necrosis imaging.

\section{Discovery of rhein as a new NAA}

The discovery of rhein as a new NAA was closely related to the research on necrosis avidity of bisanthracene nuclear anthraquinone compounds. Therefore, combing relevant background information helps to understand the discovering process of rhein as a new NAA. A schematic diagram of the discovery of necrosis avidity of rhein based on structural strategy is presented in Figure 2. Hypericin, a naturally occurring naphthodianthrone compound initially found in Hypericum perforatum L. and used as an antidepressant, antiviral and tumoricidal photosensitizer [50-53], was the first anthraquinone compound that had been found to have prominent necrosis avidity [54,55]. It has been used for determination of tissue viability ex vivo by its own intrinsic fluorescence [27,56,57] or for noninvasive visualization of myocardial necrosis as well as monitoring of treatment-induced tumor damage after being labeled with different radionuclides [58-61]. However, as a compound with polycyclic aromatic conjugated structure, hypericin is easy to form aggregates in aqueous environment, resulting in decreased targeting accumulation in necrotic tissues and unwanted uptake in mononuclear phagocyte system (MPS) organs [29,30]. Moreover, the high blood pool activity resulting from its slow plasma clearance would interfere with the visualization of myocardial necrosis, making necrotic myocardium unidentifiable until $9 \mathrm{~h}$ after injection of ${ }^{123}$ I-labeled hypericin $\left({ }^{123}\right.$ I-hypericin $)$ [58]. Therefore, it is desirable to find new NAAs with preferable physicochemical properties, pharmacokinetics and biodistribution for scintigraphic imaging.

It was reported that reducing the size of $\pi$-conjugated plane and/or distorting 
$\pi$-conjugated plane could improve aqueous solubility of small molecular compounds $[62,63]$ or could decrease or even prevent the formation of molecule aggregations $[64,65]$, thus potentially improving the physicochemical properties and biodistribution. Benzodianthrone compound protohypericin and median dianthrone compounds such as sennoside $\mathrm{A}$ and $\mathrm{B}$, sennidin $\mathrm{A}$ and $\mathrm{B}$ as well as emodin dianthrone were subsequently evaluated in animal models of induced necrosis because the former has a reduced $\pi$-conjugated core and the latter lose the nearly planar $\pi$-conjugated core in contrast to hypericin [35,37-40]. Although these compounds exhibited significantly reduced uptakes in MPS organs, they also showed reduced or even dramatically reduced targeting accumulations in necrotic tissues [35,37-40]. This initially made us to think that a rigid polycyclic aromatic conjugated structure might be the necessary structure for this kind of compounds to maintain significant necrosis avidity. With this in mind, we synthesized two more water-soluble hypericin derivatives namely hypericin dicarboxylic acid and hypericin-2,5-disulfonic acid sodium and evaluated their potential for rapid imaging of necrotic myocardium [42,43]. Although more rapid visualization of necrotic myocardium could be achieved by using ${ }^{131}$ I-hypericin dicarboxylic acid (6 h after injection) or ${ }^{131}$ I-hypericin-2,5-disulfonic acid sodium (4 h after injection) than by using ${ }^{131}$ I-hypericin, a comparable $\left({ }^{131}\right.$ I-hypericin dicarboxylic acid) or even higher $\left({ }^{131}\right.$ I-hypericin-2,5-disulfonic acid sodium) liver uptake was observed in comparison to ${ }^{131}$ I-hypericin $[42,43]$, which was still an interference with the accurate visualization of necrotic myocardium.

On the other hand, in our attempts to find alternative NAAs, skyrin, a dianthraquinone compound consisted of two molecules of emodin coupled by a single bond, was found to exhibit not only a comparable necrosis avidity to hypericin but also a dramatically reduced self-aggregation capacity [41]. However, skyrin appeared to be unsuitable for necrosis imaging because it exhibited a significantly higher blood pool activity than hypericin [41]. Nevertheless, the discovery of necrosis avidity of skyrin further deepened our understanding of the structural requirements of NAAs and inspired our exploration of monomeric anthraquinone compounds with the hope of finding new NAAs with better physicochemical properties, pharmacokinetics and 
biodistribution.

Eight monomeric anthraquinones were selected to radiolabel with ${ }^{131} \mathrm{I}$ and were preliminarily evaluated for their necrosis avidity in a mouse model of induced muscular necrosis [44]. All eight ${ }^{131}$ I-labeled anthraquinones $\left({ }^{131} \mathrm{I}\right.$-anthraquinones) were found to have significantly higher uptakes in necrotic muscle than in viable muscle by comparing the results of autoradiography and triphenyltetrazolium chloride (TTC) staining [44]. Radioactivity quantitative study showed that ${ }^{131}$ I-rhein (Figure 1) exhibited higher uptake in necrotic muscle as well as higher uptake ratio between necrotic and viable muscle than other seven ${ }^{131}$ I-anthraquinones [44]. Biodistribution study indicated that ${ }^{131}$ I-rhein had a significant clearance from normal organs within $24 \mathrm{~h}$ except for the excretory organs such as liver and kidneys [44]. Pharmacokinetics study demonstrated that ${ }^{131}$ I-rhein exhibited a blood elimination half-life of $8.20 \mathrm{~h}$ [44], which was significantly shorter than that of ${ }^{131}$ I-hypericin $[42,43]$. These results showed that monomeric anthraquinone compounds still retained necrosis avidity comparable to that of hypericin, and rhein could serve as a new necrosis-avid lead compound.

\section{Noninvasive visualization of myocardial necrosis}

The discovery of necrosis avidity of rhein has expanded its use for imaging of myocardial necrosis. In a rat model with reperfused AMI, we first explored the potential of ${ }^{131}$ I-rhein for rapid visualization of myocardial necrosis [44]. Single photon emission computed tomography/computed tomography (SPECT/CT) imaging demonstrated that an obvious hotspot was observed in the heart of rat with AMI but not in the heart of rat with sham operation at $6 \mathrm{~h}$ after administration of ${ }^{131}$ I-rhein (Figure 3A) [44]. Postmortem histopathological examination and autoradiography study showed that ${ }^{131}$ I-rhein was mainly concentrated in necrotic myocardium (Figure 3C), with an uptake ratio of up to 14 times between necrotic and viable myocardium [44]. These results suggested that the obvious hotspot observed in the heart of model rat in SPECT/CT image was resulted from the selective uptake of ${ }^{131}$ I-rhein by necrotic myocardium, or in other words, ${ }^{131}$ I-rhein was capable of non-invasively 
imaging necrotic myocardium by SPECT/CT scan [44]. Moreover, we found that it was impossible for ${ }^{131}$ I-hypericin to visualize necrotic myocardium by SPECT/CT imaging at $6 \mathrm{~h}$ after administration due to the high blood pool activity $[42,44]$. This highlighted the potential of rhein superior to hypericin as a new NAA for cardiac nuclear imaging.

In order to develop a necrosis-avid molecular probe that is more suitable for clinical diagnosis, ${ }^{99 \mathrm{~m}} \mathrm{Tc}$ instead of ${ }^{131} \mathrm{I}$ was used for labeling rhein [45]. Three ${ }^{99 \mathrm{~m}} \mathrm{Tc}$-labeled rhein derivatives (Figure 1) were synthesized and evaluated in mouse models of induced muscular necrosis [45]. Although all three ${ }^{99 \mathrm{~m}} \mathrm{Tc}$-labeled rhein derivatives exhibited significantly higher uptakes in necrotic muscle than in viable muscle and comparable necrotic-to-viable tissue uptake ratios, ${ }^{99 \mathrm{~m}} \mathrm{Tc}(\mathrm{EDDA})-\mathrm{HYNIC}-2 \mathrm{C}$-rhein presented much lower uptake in liver and spleen [45]. Thus, ${ }^{99 m} \mathrm{Tc}(\mathrm{EDDA})-\mathrm{HYNIC}-2 \mathrm{C}$-rhein was selected for further imaging study in a rat model with reperfused AMI. SPECT/CT imaging study demonstrated that an obvious hotspot was observed in the myocardium of model rat at $1 \mathrm{~h}$ post injection of ${ }^{99 m} \mathrm{Tc}($ EDDA)-HYNIC-2C-rhein while no any meaningful uptake was observed in the myocardium of rat with sham operation at the same time point (Figure 3B) [45]. Postmortem histopathological examination and autoradiography study manifested that ${ }^{99 \mathrm{~m}} \mathrm{Tc}(\mathrm{EDDA})-\mathrm{HYNIC}-2 \mathrm{C}$-rhein specifically localized in the necrotic myocardium with a 4.9-fold necrotic-to-viable myocardium ratio [45]. These results support the potential use of ${ }^{99 \mathrm{~m}} \mathrm{Te}(\mathrm{EDDA})-\mathrm{HYNIC}-2 \mathrm{C}$-rhein for rapid imaging of necrotic myocardium.

\section{Early monitoring of tumor response to therapy}

The favorable performance of ${ }^{99 \mathrm{~m}} \mathrm{Tc}(\mathrm{EDDA})$-HYNIC-2C-rhein for rapidly imaging myocardial necrosis has promoted its use for early monitoring of tumor response to therapy. In vitro cell binding assay showed that ${ }^{99 \mathrm{~m}} \mathrm{Tc}(\mathrm{EDDA})-\mathrm{HYNIC}-2 \mathrm{C}-$ rhein presented a significantly higher uptake in hyperthermia-induced necrotic A549 cells than in untreated A549 cells, and its uptake in the necrotic cells could be significantly blocked by HYNIC-2C-rhein, suggesting the specific avidity of 
${ }^{99 m} \mathrm{Tc}($ EDDA)-HYNIC-2C-rhein for necrotic cells [46]. In a rat model bearing orthotopic liver W256 tumor, microwave ablation (MWA) therapy-induced tumor necrosis could be non-invasively visualized by SPECT/CT imaging at $4 \mathrm{~h}$ after injection of ${ }^{99 m} \mathrm{Tc}(\mathrm{EDDA})-\mathrm{HYNIC}-2 \mathrm{C}$-rhein [46]. In a subcutaneous W256 tumor-bearing rat model treated with combretastatin A-4 disodium phosphate (CA4P) for $24 \mathrm{~h}$, an obvious hotspot was observed in the tumor location at $5 \mathrm{~h}$ following injection of ${ }^{99 \mathrm{~m}} \mathrm{Tc}(\mathrm{EDDA})-H Y N I C-2 \mathrm{C}$-rhein, while no uptake was visible in the untreated tumor [46]. Moreover, it was demonstrated that the uptake of ${ }^{99 \mathrm{~m}} \mathrm{Tc}(\mathrm{EDDA})-\mathrm{HYNIC}-2 \mathrm{C}$-rhein in CA4P-treated tumor could be dramatically reduced by pre-injection of HYNIC-2C-rhein [46]. Postmortem histopathological examination and autoradiography study showed that CA4P treatment led to significantly increased tumor necrosis and the uptake of ${ }^{99 \mathrm{~m}} \mathrm{Tc}(\mathrm{EDDA})-\mathrm{HYNIC}-2 \mathrm{C}$-rhein was mainly located in the necrotic area within tumors [46]. These results suggested that ${ }^{99 \mathrm{~m}} \mathrm{Tc}(\mathrm{EDDA})-\mathrm{HYNIC}-2 \mathrm{C}-$-rhein was able to reliably monitor treatment-induced tumor necrosis.

Considering the higher spatial resolution and free of ionizing radiation with magnetic resonance imaging (MRI) relative to nuclear imaging, rhein-based MRI contrast agents have been developed and studied for early monitoring tumor response to therapy [47]. Three gadolinium complexes of rhein, named $\mathrm{GdL}_{1}, \mathrm{GdL}_{2}$, and $\mathrm{GdL}_{3}$ (Figure 1), were synthesized by conjugating rhein with gadolinium 2-[4,7,10-tris(carboxymethyl)-1,4,7,10-tetraazacyclododec-1-yl]acetic acid (Gd-DOTA) through differently lengthy linkers [47]. All the three contrast agents exhibited significantly higher longitudinal relaxivities than the commercially available Gd-DOTA [47]. Moreover, they showed high thermodynamic and kinetic stability in vitro as well as negligible cytotoxicity to A549 cells in the concentration range from $0.025 \mathrm{mM}$ to $1.0 \mathrm{mM}$ [47]. As $\mathrm{GdL}_{1}$ presented the highest water solubility among them, it was selected for further study [47]. In vitro MRI showed significantly higher signal intensities in hyperthermia-induced necrotic A549 and HepG2 cells relative to untreated counterparts after being incubated with $\mathrm{GdL}_{1}$ for $30 \mathrm{~min}$, suggesting higher uptake of $\mathrm{GdL}_{1}$ both in necrotic A549 and HepG2 cells than in untreated counterparts 
[47]. In vivo MRI in rat models with induced liver necrosis showed that the necrotic liver presented obvious hyperintensity relative to normal liver at 0.5 to $12 \mathrm{~h}$ post injection of $\mathrm{GdL}_{1}$, with maximum necrotic-to-normal liver contrast appearing at $3 \mathrm{~h}$ post injection, which was consistent with the results of biodistribution in the same rat models after administration of ${ }^{64} \mathrm{CuL}_{1}\left(\mathrm{Gd}^{3+}\right.$ was replaced by ${ }^{64} \mathrm{Cu}$ for biodistribution analysis) [47]. Moreover, it was demonstrated that $\mathrm{GdL}_{1}$ presented a better imaging contrast between necrotic and normal liver as well as a longer imaging time window compared to Gd-DOTA [47]. All these results indicated that $\mathrm{GdL}_{1}$ as a necrosis-specific contrast agent was superior to Gd-DOTA, a typical non-specific extracellular contrast agent [47]. Thus, $\mathrm{GdL}_{1}$ was further explored for its potential in early monitoring of tumor response to therapy. MRI in a W256-bearing rat model demonstrated that tumor necrosis induced by CA4P treatment could be significantly enhanced and the boundary of tumor necrosis was clearly visible at $3 \mathrm{~h}$ after injection of $\mathrm{GdL}_{1}$ (Figure 4A) [47]. Therefore, $\mathrm{GdL}_{1}$ may serve as a potential MRI contrast agent for early evaluation of tumor response to necrosis-inducing therapies.

The potential of these above-mentioned rhein-based MRI contrast agents for early monitoring of treatment response was further explored in rat models with liver MWA or liver tumor MWA. We first further examined the cytotoxicity of $\mathrm{GdL}_{1}, \mathrm{GdL}_{2}$, and $\mathrm{GdL}_{3}$ in $\mathrm{HepG} 2$ cells by the MTT (3-(4,5-dimethyl-2-thiazolyl)-2,5-diphenyl-2-H-tetrazolium bromide) assay [48]. The results demonstrated that $\mathrm{GdL}_{3}$ was not as safe as $\mathrm{GdL}_{1}$ and $\mathrm{GdL}_{2}$ in the concentration range from 25 to $1000 \mu \mathrm{M}$ [48]. Further comparison of $\mathrm{GdL}_{1}$ and $\mathrm{GdL}_{2}$ for imaging MWA-induced necrotic lesion in a rat model of liver MWA showed that much clearer visualization of the MWA lesion was observed both at 3 and $6 \mathrm{~h}$ post injection of $\mathrm{GdL}_{2}$ in contrast to $\mathrm{GdL}_{1}$ [48]. Thus, $\mathrm{GdL}_{2}$ was selected for further evaluation. It was demonstrated that $\mathrm{GdL}_{2}$ could enhance the ablated lesion in rats with liver MWA as early as $2 \mathrm{~h}$ post ablation and could maintain a stable contrast ratio of ablated lesion to normal liver until $24 \mathrm{~h}$ post ablation, which supported its potential use for early assessment of thermal ablation therapy response [48]. Then the capability of $\mathrm{GdL}_{2}$ to distinguish residual tumor from MWA lesion was evaluated in a rat model of liver 
tumor with intentionally incomplete MWA. It was demonstrated that $\mathrm{GdL}_{2}$ could selectively enhance MWA-induced necrotic tumor while rendering residual viable tumor hyperintensity relative to normal liver at $3 \mathrm{~h}$ post injection (Figure 4B), thus allowing noninvasive identification of unaffected residual tumor [48]. Moreover, it was found that the optimal time window for visualizing the MWA lesion appeared at 2.5 to $3.5 \mathrm{~h}$ post injection of $\mathrm{GdL}_{2}$ in rat models of liver tumor MWA [48]. In the subsequent comparative study of $\mathrm{GdL}_{2}$ and Gd-DOTA for the therapeutic evaluation of MWA, $\mathrm{GdL}_{2}$ showed significantly better contrast-enhanced imaging performance than Gd-DOTA [48]. All these results indicate that $\mathrm{GdL}_{2}$ has a considerable potential in early monitoring of tumor response to MWA [48].

Considering the significantly higher sensitivity and acceptable spatial resolution of positron emission tomography (PET) imaging compared to MRI, a rhein-based PET probe $\left({ }^{68} \mathrm{Ga}\right.$-DOTA-rhein, Figure 1) has been developed by substituting the $\mathrm{Gd}^{3+}$ in $\mathrm{GdL}_{2}$ with positron radionuclide ${ }^{68} \mathrm{Ga}^{3+}$ for early monitoring of therapy-induced tumor necrosis [49]. The initial biodistribution study in a mouse model of induced muscular necrosis demonstrated that ${ }^{68}$ Ga-DOTA-rhein exhibited a significantly higher uptake in necrotic muscle than in normal muscle at both 30 and 60 min post injection [49]. Autoradiography and TTC staining studies further confirmed the results of biodistribution, revealing the specific avidity of ${ }^{68} \mathrm{Ga}$-DOTA-rhein for necrotic tissues [49]. Micro-PET/CT imaging showed that an obvious hotspot was observed in the necrotic region of the left hind limb but not in the right normal hind limb at $1 \mathrm{~h}$ post injection of ${ }^{68} \mathrm{Ga}$-DOTA-rhein, suggesting that ${ }^{68} \mathrm{Ga}$-DOTA-rhein could be used for visualization of necrotic tissues in vivo [49]. In a S180 tumor-bearing mouse model, PET/CT imaging showed significantly higher radioactive uptake in the CA4P-treated tumors compared to the untreated tumors at $1 \mathrm{~h}$ post injection of ${ }^{68} \mathrm{Ga}$-DOTA-rhein [49]. The result of ex vivo biodistribution was consistent with the finding of PET/CT imaging [49]. The result of autoradiography further confirmed the higher uptake of ${ }^{68} \mathrm{Ga}$-DOTA-rhein in the CA4P-treated tumors than in the untreated tumors, which was closely related to the increased tumor necrosis induced by CA4P [49]. All these results indicated that ${ }^{68} \mathrm{Ga}$-DOTA-rhein could be used for early monitoring of tumor 
necrotic response.

\section{Mechanism exploration of necrosis avidity behind rhein}

The exposure of intracellular DNA is a common hallmark of cell necrosis $[1,66]$. Exposed DNA can be targeted for developing molecular imaging probes that are specific to necrotic tissues [67-73]. As anthraquinone compounds are a well-known class of DNA intercalators $[74,75]$, thus we speculated that the necrosis avidity of rhein might be resulted from its binding to exposed DNA in necrotic tissues. In vitro DNA binding studies demonstrated both rhein and its derivatives could bind to DNA in an intercalative manner with binding constants and quenching constants ranging in order of magnitude of $10^{4} \mathrm{M}^{-1}[45,47,49,76]$, which fall within the typical range $\left(10^{4}\right.$ $10^{6} \mathrm{M}^{-1}$ ) of binding constants that have been reported for compounds that bind to DNA by intercalation mode [77].

In order to examine the subcellular distribution of rhein, a clickable rhein-azide derivative was synthesized by introduction of an azide group at the position of carboxyl group of rhein, which retained the necrosis avidity similar to rhein and was amenable to bioorthogonal fluorescent labeling using click-chemistry (Figure 5A) [76]. In vitro cell binding studies indicated that both ${ }^{99 \mathrm{~m}} \mathrm{Tc}(\mathrm{EDDA})-\mathrm{HYNIC}-2 \mathrm{C}$-rhein and ${ }^{131}$ I-labeled rhein-azide ( ${ }^{131}$ I-rhein-azide) exhibited significantly higher uptake in necrotic A549 cells than in viable A549 cells [46,76]. Although both ${ }^{131}$ I-rhein and ${ }^{131}$ I-rhein-azide presented relatively higher uptakes in necrotic red cells than in viable red cells, their uptakes in necrotic red cells were significantly lower than those in necrotic A549 cells [76]. Considering that mature red cells are free of nucleus, the significantly higher uptake of rhein derivatives in necrotic A549 cells than in necrotic red cells might be closely related to the presence of the nucleus in necrotic A549 cells [76]. Cellular colocalization studies demonstrated that rhein-azide mainly accumulated in the nucleus and nucleolus of necrotic A549 cells while not in viable A549 cells (Figure 5B) [76]. As we know, the nucleus is the main existing place of intracellular DNA, which becomes accessible to binding by molecular probes after the rupture of cell plasma membrane integrity. Based on the above experiments, we 
concluded that the specific avidity of rhein and its derivatives for necrotic cells might be due to their binding to exposed intracellular DNA.

Furthermore, it was found that the uptakes of radiolabeled rhein derivatives in necrotic tissues could be significantly reduced by rhein itself or its unlabeled rhein derivatives, suggesting that the uptakes of rhein and its derivatives in necrotic tissues were target-specific $[45,46,76]$. Propidium iodide as a membrane-impermeable DNA staining reagent, which releases red fluorescence after insertion of double-stranded DNA, has long been used as a marker for necrosis in flow cytometry. In our studies, in vivo propidium iodide staining was used to verify DNA exposure in necrotic tissues $[46,76]$. It was demonstrated that a significantly higher red fluorescence intensity released by propidium iodide was observed in necrotic tissues than in normal tissues and that propidium iodide was mainly located in the nuclei of necrotic cells, which indicated that the loss of plasma membrane integrity of necrotic cells allowed the free access of propidium iodide to intracellular DNA, or in other words, the intracellular DNA became exposed in necrotic tissues [46,76]. In summary, we concluded that the necrosis avidity of rhein and its derivatives was to a large extent resulted from their binding to exposed DNA in necrotic tissues.

\section{Conclusions}

It has been more than 45 years since the first discovery of necrosis avidity of small molecular compounds. Rhein as a naturally occurring monomeric anthraquinone compound was recently demonstrated to have a newly discovered necrosis-avid trait, which has driven the development of rhein-based necrosis-avid molecular probes and their applications in necrosis imaging. By taking advantage of rhein-based molecular probes, rapid visualization of necrotic myocardium as well as early monitoring of tumor response to therapy could be successfully achieved. The necrosis-avid mechanism of rhein-based molecular probes was in a large part attributed to their binding to exposed DNA in necrotic tissues. Overall, the discovery of necrosis avidity of rhein and the development of rhein-based necrosis-avid molecular probes have further expanded the scope of NAAs and highlighted the potential utility of NAAs in 
necrosis imaging.

\section{Future perspectives}

Although currently developed rhein-based molecular probes have shown promising potential for necrosis imaging, further evaluation and structural optimizations of rhein-based molecular probes are still necessary. For instance, in view of the high spatial resolution and free of ionizing radiation of MRI technology, rhein-based MRI contrast agents are expected to further expand their imaging applications in other necrosis-related diseases such as AMI and acute pancreatitis. However, due to the low sensitivity of MRI technology, a significantly higher dose of contrast agents compared to molecular probes used in SPECT or PET imaging is generally administrated for the contrast enhancement of MRI, which undoubtedly increases the water solubility requirement of MRI contrast agents. Moreover, $\mathrm{Gd}^{3+}$-based contrast agents may be subjected to potential toxicity risks $[78,79]$. Therefore, further structural optimizations to increase the relaxivities and/or increase the stability of rhein-based $\mathrm{Gd}^{3+}$-chelated MRI contrast agents are desirable, which help to reduce the dosage and/or the potential risks of these contrast agents [80]. On the other hand, considering the facts that PET is capable of offering well-defined, three-dimensional quantitative images of the biodistribution of molecular probes and has a higher sensitivity than other molecular imaging techniques, and that ${ }^{18} \mathrm{~F}$ can provide better spatial resolution for PET imaging than other positron emitters and enable lower radiation exposure to patients due to its unique properties $[81,82]$, it could be an advisable alternative to construct rhein-based ${ }^{18} \mathrm{~F}$-labeled PET molecular probes in the future studies.

\section{Acknowledgments}

This work was supported in part by the National Natural Science Foundation of China [81771870 and 21907053]; the Natural Science Foundation of Jiangsu province [20171512]; the Six Talent Peaks Project of Jiangsu Province [YY-011]; and the "Six one project" for high level medical talents [LGY2018075].

\section{Disclosure statement}

The authors report no conflict of interest. 


\section{References}

[1] Zhang D, Gao M, Jin Q, et al. Updated developments on molecular imaging and therapeutic strategies directed against necrosis. Acta Pharm Sin B. 2019;9(3):455-468.

[2] Miranda Cona M, Oyen R, Ni Y. Necrosis avidity of organic compounds: a natural phenomenon with exploitable theragnostic potentials. Curr Med Chem. 2015;22(15):1829-1849.

[3] Bonte FJ, Parkey RW, Graham KD, et al. A new method for radionuclide imaging of myocardial infarcts. Radiology. 1974;110(2):473-474.

[4] Ni Y. Metalloporphyrins and functional analogues as MRI contrast agents. Curr Med Imaging Rev. 2008;4(2):96-112.

[5] Ni Y, Bormans G, Chen F, et al. Necrosis avid contrast agents: functional similarity versus structural diversity. Invest Radiol. 2005;40(8):526-535.

[6] Jiang B, Wang J, Ni Y, et al. Necrosis avidity: a newly discovered feature of hypericin and its preclinical applications in necrosis imaging. Theranostics. 2013;3(9):667-676.

[7] Li J, Oyen R, Verbruggen A, et al. Small molecule sequential dual-targeting theragnostic strategy (SMSDTTS): from preclinical experiments towards possible clinical anticancer applications. J Cancer. 2013;4(2):133-145.

[8] Cona MM, de Witte P, Verbruggen A, et al. An overview of translational (radio) pharmaceutical research related to certain oncological and non-oncological applications. World J Methodol. 2013;3(4):45-64.

[9] De Saint-Hubert M, Bauwens M, Deckers N, et al. In vivo molecular imaging of apoptosisand necrosis in atherosclerotic plaquesusing microSPECT-CT and microPET-CT imaging. Mol Imaging Biol. 2014;16(2):246-254

[10] Fang C, Wang K, Zeng C, et al. Illuminating necrosis: From mechanistic exploration to preclinical application using fluorescence molecular imaging with indocyanine green. Sci Rep. 2016;6:21013.

[11] Xie B, Stammes MA, van Driel PBAA, et al. Necrosis avid near infrared 
fluorescent cyanines for imaging cell death and their use to monitor therapeutic efficacy in mouse tumor models. Oncotarget. 2015;6(36):39036-39049.

[12] Stammes MA, Maeda $\mathrm{A}, \mathrm{Bu}$ J, et al. The necrosis-avid small molecule HQ4-DTPA as a multimodal imaging agent for monitoring radiation therapy-induced tumor cell death. Front Oncol. 2016;6:221.

[13] Stammes MA, Knol-Blankevoort VT, Cruz LJ, et al. Pre-clinical evaluation of a cyanine-based SPECT probe for multimodal tumor necrosis imaging. Mol Imaging Biol. 2016;18(6):905-915.

[14] Zhu M, Lin XA, Zha XM, et al. Evaluation of the therapeutic efficacy of sequential therapy involving percutaneous microwave ablation in combination with ${ }^{131}$ I-hypericin using the VX2 rabbit breast solid tumor mode. PLoS One. 2015;10:e0120303.

[15] Jendželovská Z, Jendželovský R, Kuchárová B, et al. Hypericin in the light and in the dark: two sides of the same coin. Front Plant Sci. 2016;7:560.

[16] Rybczynska AA, Boersma HH, de Jong S, et al. Avenues to molecular imaging of dying cells: Focus on cancer. Med Res Rev. 2018;38(6):1713-1768.

[17] Li Y, Wang S, Zhao Y, et al. A model in vitro study using hypericin:tumor-versus necrosis-targeting property and possible mechanisms. Biology. 2020;9(1):13.

[18] Zhou YX, Xia W, Yue W, et al. Rhein: a review of pharmacological activities. Evid Based Complement Alternat Med. 2015;2015:578107.

[19] Sun H, Luo G, Chen D, et al. A comprehensive and system review for the pharmacological mechanism of action of rhein, an active anthraquinone ingredient. Front Pharmacol. 2016;7:247.

[20] Wu C, Cao H, Zhou H, et al. Research progress on the antitumor effects of rhein: literature review. Anticancer Agents Med Chem. 2017;17(12):1624-1632.

[21] Li J, Sun Z, Zhang J, et al. A dual-targeting anticancer approach: soil and seed principle. Radiology. 2011;260(3):799-807.

[22] Li J, Cona MM, Chen F, et al. Exploring theranostic potentials of radioiodinated hypericin in rodent necrosis models. Theranostics. 2012;2(10):1010-1019.

[23] Li J, Cona MM, Chen F, et al. Sequential systemic administrations of 
combretastatin A4 Phosphate and radioiodinated hypericin exert synergistic targeted theranostic effects with prolonged survival on SCID mice carrying bifocal tumor xenografts. Theranostics. 2013;3(2):127-137.

[24] Kong M, Zhang J, Jiang C, et al. Necrosis affinity evaluation of ${ }^{131}$ I-hypericin in a rat model of induced necrosis. J Drug Target. 2013;21(6):604-610.

[25] Ji Y, Zhan Y, Jiang C, et al. Improvement of solubility and targetability of radioiodinated hypericin by using sodium cholate based solvent in rat models of necrosis. J Drug Target. 2014;22(4):304-312.

[26] Cona MM, Feng Y, Verbruggen A, et al. Improved clearance of radioiodinated hypericin as a targeted anticancer agent by using a duodenal drainage catheter in rats. Exp Biol Med. 2013;238(12):1437-1449.

[27] Jiang C, Li Y, Jiang X, et al. Hypericin as a marker for determination of myocardial viability in a rat model of myocardial infarction. Photochem Photobiol. 2014;90(4):867-872.

[28] Qi X, Shao H, Zhang J, et al. Radiopharmaceutical study on Iodine-131-labelled hypericin in a canine model of hepatic RFA-induced coagulative necrosis. Radiol Med. 2015;120(2):213-221.

[29] Cona MM, Alpizar YA, Li J, et al. Radioiodinated hypericin: its biodistribution, necrosis avidity and therapeutic efficacy are influenced by formulation. Pharm Res. 2014;31(2):278-290.

[30] Liu X, Jiang C, Li Y, et al. Evaluation of hypericin: effect of aggregation on targeting biodistribution. J Pharm Sci. 2015;104(1):215-222.

[31] Ji Y, Jiang C, Zhang X, et al. Necrosis targeted combinational theragnostic approach to treat cancer. Oncotarget. 2014;5(10):2934-2946.

[32] Cona MM, Feng Y, Zhang J, et al. Sodium cholate, a solubilizing agent for the necrosis avid radioiodinated hypericin in rabbits with acute myocardial infarction. Drug Deliv. 2015;22(3):427-435.

[33] Liu W, Zhang D, Feng Y, et al. Biodistribution and anti-tumor efficacy of intratumorally injected necrosis-avid theranostic agent radioiodinated hypericin in rodent tumor models. J Drug Target. 2015;23(4):371-379. 
[34] Shao H, Zhang J, Sun Z, et al. Necrosis targeted radiotherapy with iodine-131-labeled hypericin to improve anticancer efficacy of vascular disrupting treatment in rabbit VX2 tumor models. Oncotarget. 2015;6(16):14247-14259.

[35] Liu X, Feng Y, Jiang C, et al. Radiopharmaceutical evaluation of ${ }^{131}$ I-protohypericin as a necrosis avid compound. J Drug Target. 2015;23(5):417-426.

[36] Liu X, Jiang C, Zhang D, et al. Tumor necrosis targeted radiotherapy of non-small cell lung cancer using radioiodinated protohypericin in a mouse model. Oncotarget. 2015;6(28):26400-26410.

[37] Jiang C, Gao M, Li Y, et al. Exploring diagnostic potentials of radioiodinated sennidin $\mathrm{A}$ in rat model of reperfused myocardial infarction. Int $\mathrm{J}$ Pharm. 2015;495(1):31-40.

[38] Zhang D, Huang D, Ji Y, et al. Experimental evaluation of radioiodinated sennoside B as a necrosis-avid tracer agent. J Drug Target. 2015;23(2):180-190.

[39] Zhang D, Jiang C, Yang S, et al. Effects of skeleton structure on necrosis targeting and clearance properties of radioiodinated dianthrones. J Drug Target. 2016;24(6):566-577.

[40] Li L, Zhang D, Yang S, et al. Effects of glycosylation on biodistribution and imaging quality of necrotic myocardium of iodine-131-labeled sennidins. Mol Imaging Biol. 2016;18(6):877-886.

[41] Wang C, Jin Q, Yang S, et al. Synthesis and evaluation of ${ }^{131}$ I-skyrin as a necrosis avid agent for potential targeted radionuclide therapy of solid tumors. Mol Pharmaceutics. 2016;13(1):180-189.

[42] Li J, Zhang J, Yang S, et al. Synthesis and preclinical evaluation of radioiodinated hypericin dicarboxylic acid as a necrosis avid agent in rat models of induced hepatic, muscular, and myocardial necroses. Mol Pharmaceutics. 2016;13(1):232-240.

[43] Duan X, Yin Z, Jiang C, et al. Radioiodinated hypericin disulfonic acid sodium salts as a DNA-binding probe for early imaging of necrotic myocardium. Eur $\mathbf{J}$ 
Pharm Biopharm. 2017;117:151-159.

[44] Wang Q, Yang S, Jiang C, et al. Discovery of radioiodinated monomeric anthraquinones as a novel class of necrosis avid agents for early imaging of necrotic myocardium. Sci Rep. 2016;6:21341.

[45] Luo Q, Jin Q, Su C, et al. Radiolabeled rhein as small-molecule necrosis avid agents for imaging of necrotic myocardium. Anal Chem. 2017;89(2):1260-1266.

[46] Liang J, Luo Q, Zhang D, et al. SPECT imaging of treatment-related tumor necrosis using technetium-99m-labeled rhein. Mol Imaging Biol. 2019;21(4):660-668.

[47] Bian L, Gao M, Zhang D, et al. Synthesis and biological evaluation of rhein-based MRI contrast agents for in vivo visualization of necrosis. Anal Chem. 2018;90(22):13249-13256.

[48] Wu T, Zhang J, Jin Q, et al. Rhein- based necrosis- avid MRI contrast agents for early evaluation of tumor response to microwave ablation therapy. Magn Reson Med. 2019;82(6):2212-2224.

[49] Zhang A, Wu T, Bian L, et al. Synthesis and evaluation of Ga-68-labeled rhein for early assessment of treatment-induced tumor necrosis. Mol Imaging Biol. 2019; DOI:10.1007/s11307-019-01365-y.

[50] Barnes J, Anderson LA, Phillipson JD. St John's wort (Hypericum perforatum L.): a review of its chemistry, pharmacology and clinical properties. J Pharm Pharmacol. 2001;53(5):583-600.

[51] Miskovsky P. Hypericin-a new antiviral and antitumor photosensitizer: mechanism of action and interaction with biological macromolecules. Curr Drug Targets. 2002;3(1):55-84.

[52] Kubin A, Wierrani F, Burner U, et al. Hypericin-the facts about a controversial agent. Curr Pharm Des. 2005;11(2):233-253.

[53] Karioti A, Bilia AR. Hypericins as potential leads for new therapeutics. Int J Mol Sci. 2010;11(2):562-594.

[54] Ni Y, Huyghe D, Verbeke K, et al. First preclinical evaluation of mono- $\left[{ }^{123} \mathrm{I}\right]$ iodohypericin as a necrosis-avid tracer agent. Eur $\mathbf{J}$ Nucl Med. 
2006;33(5):595-601.

[55] Van de Putte M, Ni Y, De Witte PAM. Exploration of the mechanism underlying the tumor necrosis avidity of hypericin. Oncol Rep. 2008;19(4):921-926.

[56] Van de Putte M, Wang H, Chen F, et al. Hypericin as a marker for determination of tissue viability after radiofrequency ablation in a murine liver tumor model. Oncol Rep. 2008;19(4):927-932.

[57] Van de Putte M, Wang H, Chen F, et al. Hypericin as a marker for determination of tissue viability after intratumoral ethanol injection in a murine liver tumor model. Acad Radiol. 2008;15(1):107-113.

[58] Fonge H, Vunckx K, Wang H, et al. Non-invasive detection and quantification of acute myocardial infarction in rabbits using mono- $\left[{ }^{123}\right.$ I] iodohypericin microSPECT. Eur Heart J. 2008;29(2):260-269.

[59] Feng Y, Cona MM, Vunckx K, et al. Detection and quantification of acute reperfused myocardial infarction in rabbits using DISA-SPECT/CT and $3.0 \mathrm{~T}$ cardiac MRI. Int J Cardiol. 2013;168(4):4191-4198.

[60] Miranda Cona M, Feng Y, Li Y, et al. Comparative study of Iodine-123-labeled-hypericin

Tc-99m-labeled-hexakis [2-methoxyisobutylisonitril] in a rabbit model of myocardial infarction. J Cardiovasc Pharm. 2013;62(3);304-311.

[61] Song S, Xiong C, Zhou M, et al. Small-animal PET of tumor damage induced by photothermal ablation with ${ }^{64} \mathrm{Cu}$-bis-DOTA-hypericin. J Nucl Med. 2011;52(5):792-799.

[62] Ishikawa M, Hashimoto Y. Improvement in aqueous solubility in small molecule drug discovery programs by disruption of molecular planarity and symmetry. $\mathbf{J}$ Med Chem. 2011;54(6):1539-1554.

[63] Lewin G, Maciuk A, Moncomble A, et al. Enhancement of the water solubility of flavone glycosides by disruption of molecular planarity of the aglycone moiety. $\mathbf{J}$ Nat Prod. 2013;76(1):8-12.

[64] Chen Z, Baumeister U, Tschierske C, et al. Effect of core twisting on self-assembly and optical properties of perylene bisimide dyes in solution and 
columnar liquid crystalline phases. Chemistry. 2007;13(2):450-465.

[65] Chen Z, Lohr A, Saha-Möller CR, et al. Self-assembled $\pi$-stacks of functional dyes in solution: structural and thermodynamic features. Chem Soc Rev. 2009;38(2):564-584.

[66]Smith BA, Smith BD. Biomarkers and molecular probes for cell death imaging and targeted therapeutics. Bioconjugate Chem. 2012;23(10):1989-2006.

[67] Garanger E, Hilderbrand SA, Blois JT, et al. A DNA-binding Gd chelate for the detection of cell death by MRI. Chem Commun. 2009;(29):4444-4446.

[68] Dasari M, Lee S, Sy J, et al. Hoechst-IR: an imaging agent that detects necrotic tissue in vivo by binding extracellular DNA. Org Lett. 2010;12(15):3300-3303.

[69] Huang S, Chen HH, Yuan H, et al. Molecular MRI of acute necrosis with a novel DNA-binding gadolinium chelate: kinetics of cell death and clearance in infarcted myocardium. Circ Cardiovasc Imaging. 2011;4(6):729-737.

[70] Cho H, Alcantara D, Yuan H, et al. Fluorochrome-functionalized nanoparticles for imaging DNA in biological systems. ACS Nano. 2013;7(3):2032-2041.

[71] Cho H, Guo Y, Sosnovik DE, et al. Imaging DNA with Fluorochrome Bearing Metals. Inorg Chem. 2013;52(21):12216-12222.

[72] Chen HH, Yuan H, Cho H, et al. Theranostic nucleic acid binding nanoprobe exerts anti-inflammatory and cytoprotective effects in ischemic injury. Theranostics. 2017;7(4):814-825.

[73] Zhang D, Gao M, Yao N, et al. Preclinical evaluation of radioiodinated Hoechst 33258 for early prediction of tumor response to treatment of vascular-disrupting agents. Contrast Media Mol Imaging. 2018;2018:5237950.

[74] Rescifina A, Zagni C, Varrica MG, et al. Recent advances in small organic molecules as DNA intercalating agents: Synthesis, activity, and modeling. Eur J Med Chem. 2014;74:95-115.

[75] Malik EM, Müller CE. Anthraquinones as pharmacological tools and drugs. Med Res Rev. 2016;36(4):705-748.

[76] Jin Q, Jiang C, Gao M, et al. Target exploration of rhein as a small-molecule necrosis avid agent by post-treatment click modification. New $\mathrm{J}$ Chem. 
2019;43(16):6121-6125.

[77] Zhang S, Sun X, Kong R, et al. Studies on the interaction of apigenin with calf thymus DNA by spectroscopic methods. Spectrochim Acta A Mol Biomol Spectrosc. 2015;136:1666-1670.

[78] Ramalho J, Semelka RC, Ramalho M, et al. Gadolinium-based contrast agent accumulation and toxicity: an update. Am J Neuroradiol. 2016;37(7):1192-1198.

[79] Kim HK, Lee GH, Chang Y. Gadolinium as an MRI contrast agent. Future Med Chem. 2018;10(6):639-661.

[80] Wahsner J, Gale E M, Rodríguez-Rodríguez A, et al. Chemistry of MRI contrast agents: current challenges and new frontiers. Chem Rev. 2018;119(2):957-1057.

[81] Gillis EP, Eastman KJ, Hill MD, et al. Applications of fluorine in medicinal chemistry. J Med Chem. 2015;58(21):8315-8359.

[82] Van Der Born D, Pees A, Poot AJ, et al. Fluorine-18 labelled building blocks for PET tracer synthesis. Chem Soc Rev. 2017;46(15):4709-4773. 


\section{Figure captions}<smiles>O=C(O)c1cc(O)c2c(c1)C(=O)c1cccc(O)c1C2=O</smiles><smiles>O=C(O)c1cc(O)c2c(c1)C(=O)c1ccc(Br)c(O)c1C2=O</smiles>

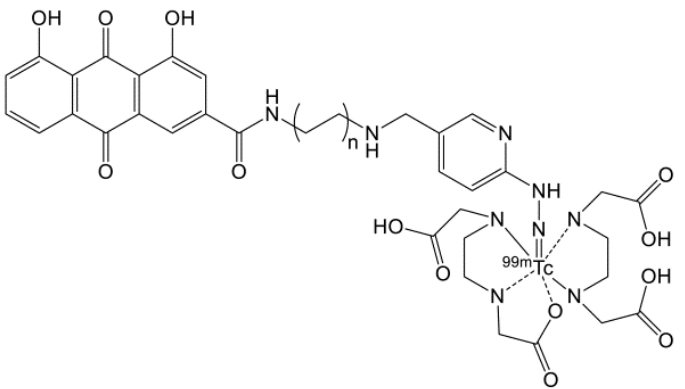

${ }^{99 m}$ Tc(EDDA)-HYNIC-2nC-Rhein, $\mathrm{n}=1$, 2, 3

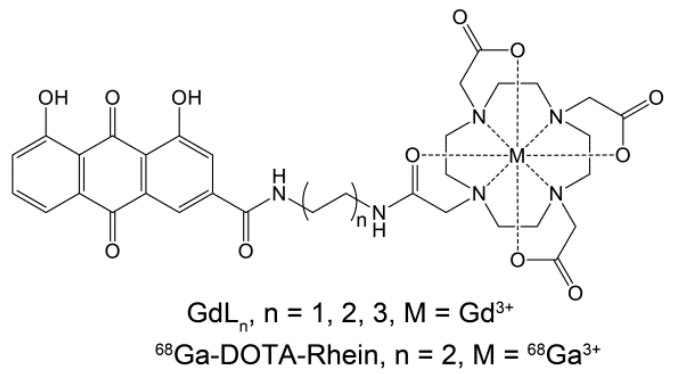

Figure 1. Chemical structures of rhein and rhein-based necrosis-avid molecular probes.
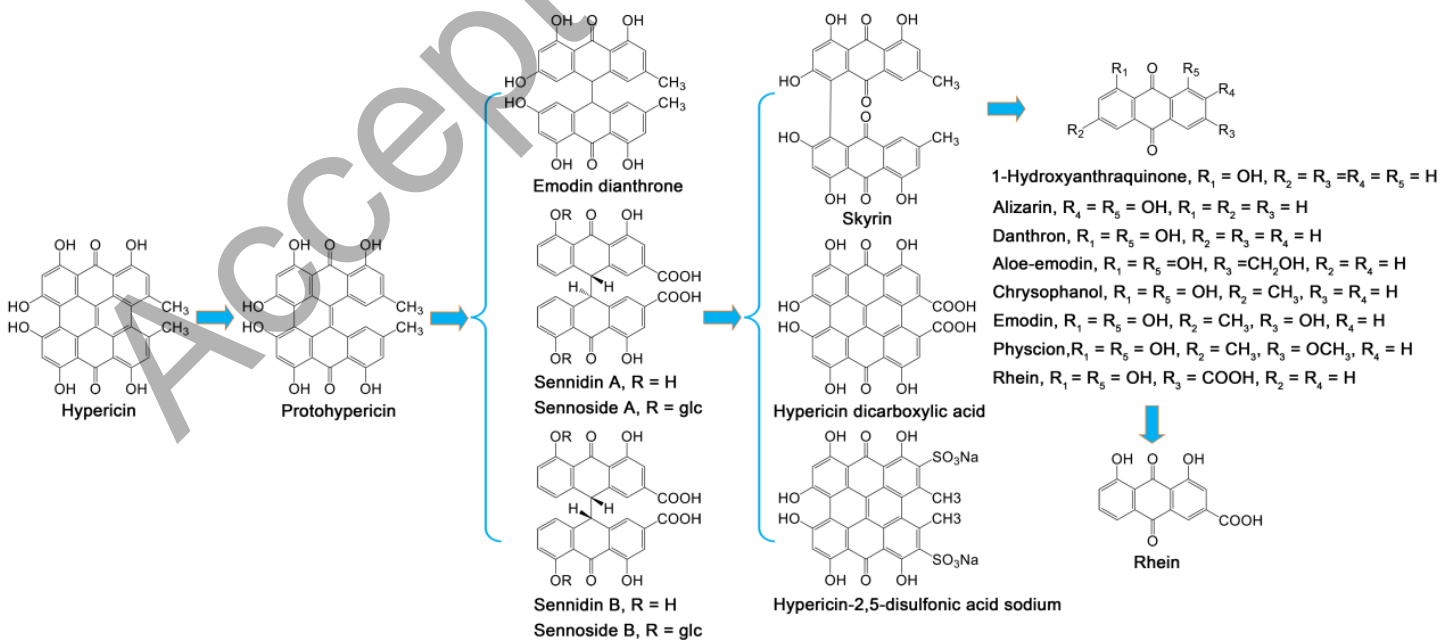

Figure 2. Schematic diagram of the discovery of necrosis avidity of rhein based on structural strategy. 


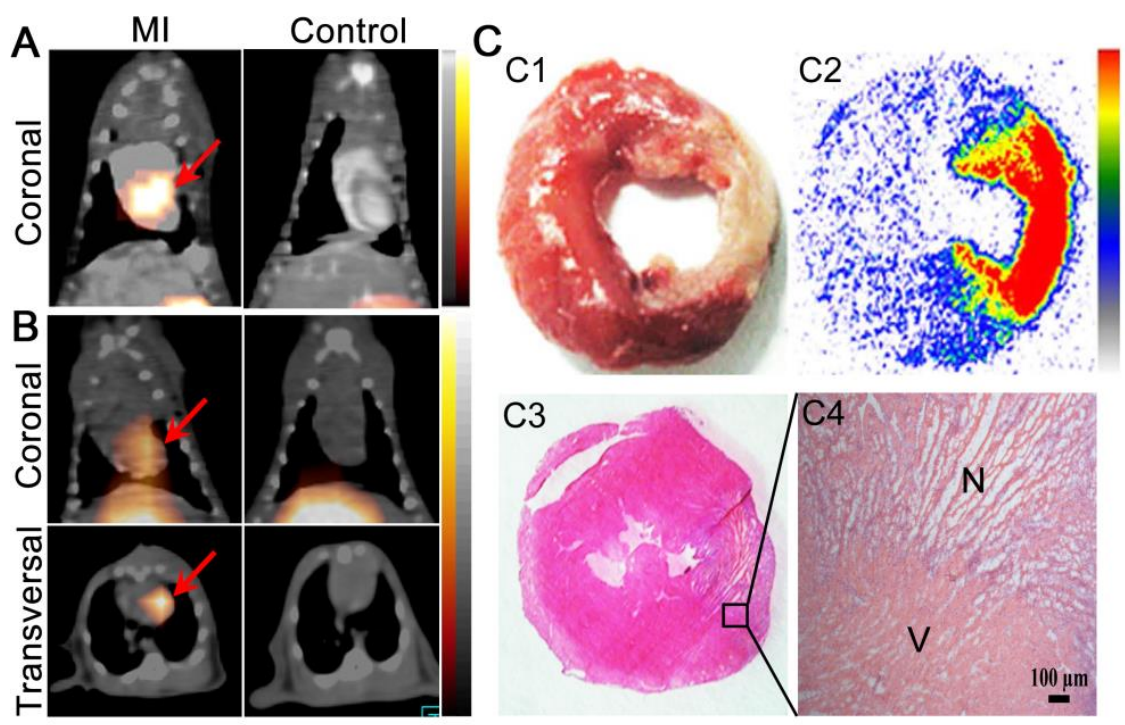

Figure 3. SPECT/CT imaging of rats with reperfused myocardium infarction (MI) or sham operation (Control) after intravenous injection of ${ }^{131}$ I-rhein (A) or ${ }^{99 m}$ Tc(EDDA)-HYNIC-2C-rhein (B). Red arrows indicate the infarcted myocardium. (C) Postmortem analysis of myocardial sections harvested from model rats injected with ${ }^{131}$ I-rhein. (C1) Photograph of $2 \mathrm{~mm}$ thick TTC-stained myocardial slice. (C2) Autoradiograph of $50 \mu \mathrm{m}$ frozen section made after TTC staining. (C3) Photograph of $10 \mu \mathrm{m}$ thick H\&E-stained myocardial section made from myocardial tissues adjacent to those used for TTC staining. (C4) Microphotograph of region of interest in C3, proving the presence of necrosis. $\mathrm{N}=$ necrotic area; $\mathrm{V}=$ viable area. Scale bar $=100$ $\mu \mathrm{m}$. (Adapted with permission from references [44,45]. Copyright ${ }^{\odot} 2016$ Springer Nature and 2017 American Chemical Society, respectively). 


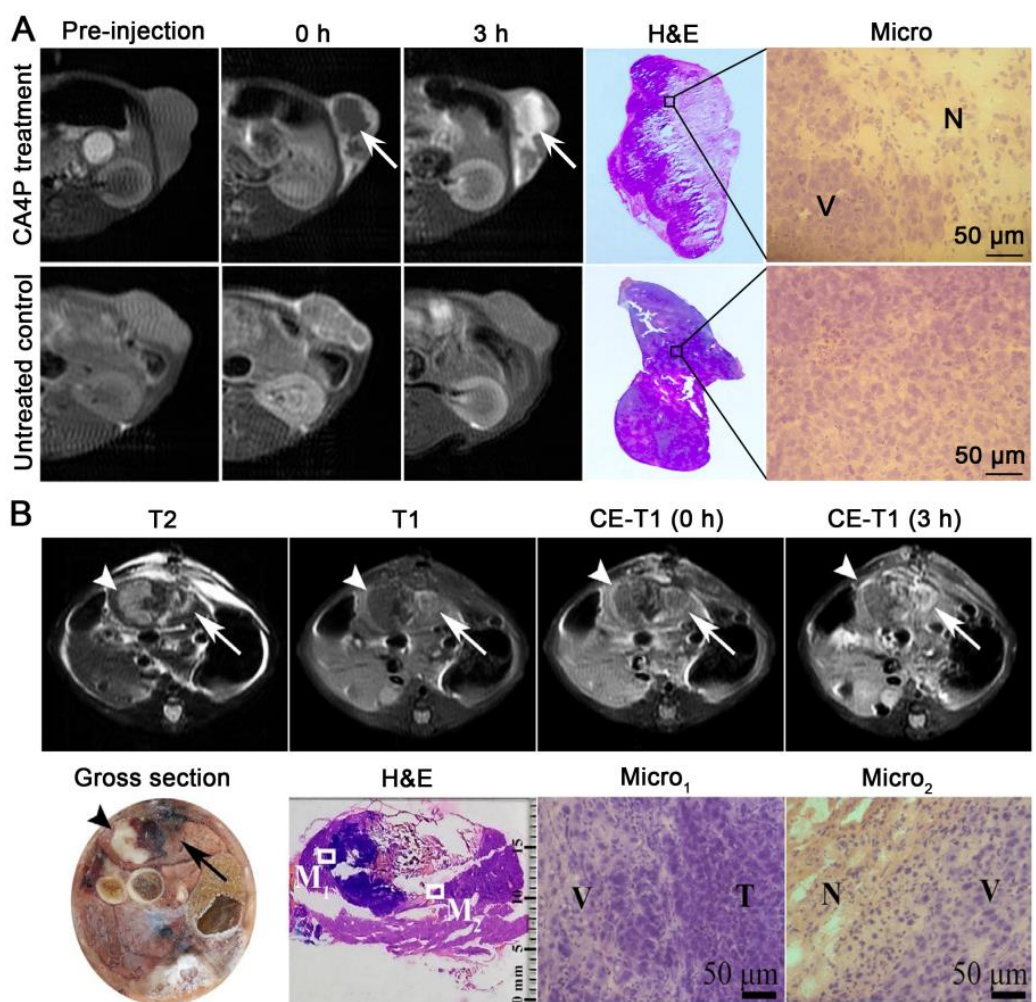

Figure 4. (A) MR images of W256 tumor-bearing rats with CA4P treatment or not at pre-injection, 0 and $3 \mathrm{~h}$ post injection of $\mathrm{GdL}_{1}$ as well as corresponding photographs of H\&E-stained tumor sections and micrographs (Micro) of regions of interest. White arrows indicate the necrotic areas of tumor. $\mathrm{N}=$ necrotic tumor; $\mathrm{V}=$ viable tumor. Scale bar $=50 \mu \mathrm{m}$. (B) Evaluation of $\mathrm{GdL}_{2}$ for distinguishing between residual tumor and MWA-induced tumor necrotic lesion. Rats bearing orthotopic liver W256 tumor subjected to intentionally incomplete MWA and received $\mathrm{GdL}_{2}$-enhanced MRI at $12 \mathrm{~h}$ after MWA treatment. T2-weighted and T1-weighted images were acquired prior to the injection of $\mathrm{GdL}_{2}$ and contrast-enhanced T1-weighted (CE-T1) images were acquired at 0 and $3 \mathrm{~h}$ post injection (upper panels). The corresponding photographs of gross section and $\mathrm{H} \& \mathrm{E}$ stained frozen section as well as micrographs $\left(\mathrm{Micro}_{1}=\mathrm{M}_{1}\right.$, $\mathrm{Micro}_{2}=\mathrm{M}_{2}$ ) of regions of interest are shown in the bottom. Arrows indicate the MWA lesion and arrowheads indicate the residual tumor. $\mathrm{N}=$ necrotic tumor; $\mathrm{V}=$ viable liver; $\mathrm{T}=$ residual tumor. Scale bar $=50 \mu \mathrm{m}$. (Adapted with permission from references [47,48]. Copyright ${ }^{\odot} 2018$ American Chemical Society and 2019 International Society for Magnetic Resonance in Medicine, respectively). 
A

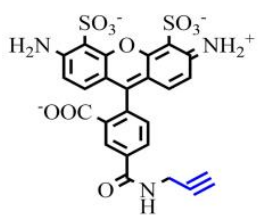

Alexa Fluor 488 alkyne
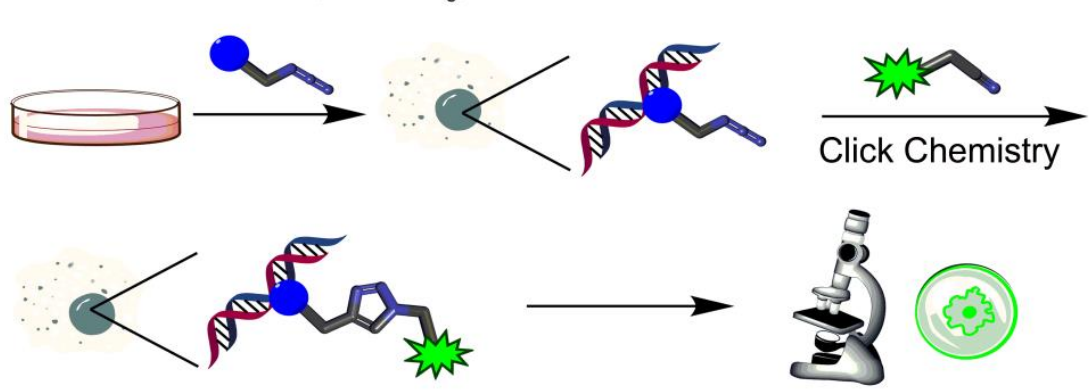

Rhein- $\mathrm{N}_{3}$ Alexa Fluor 488 alkyne

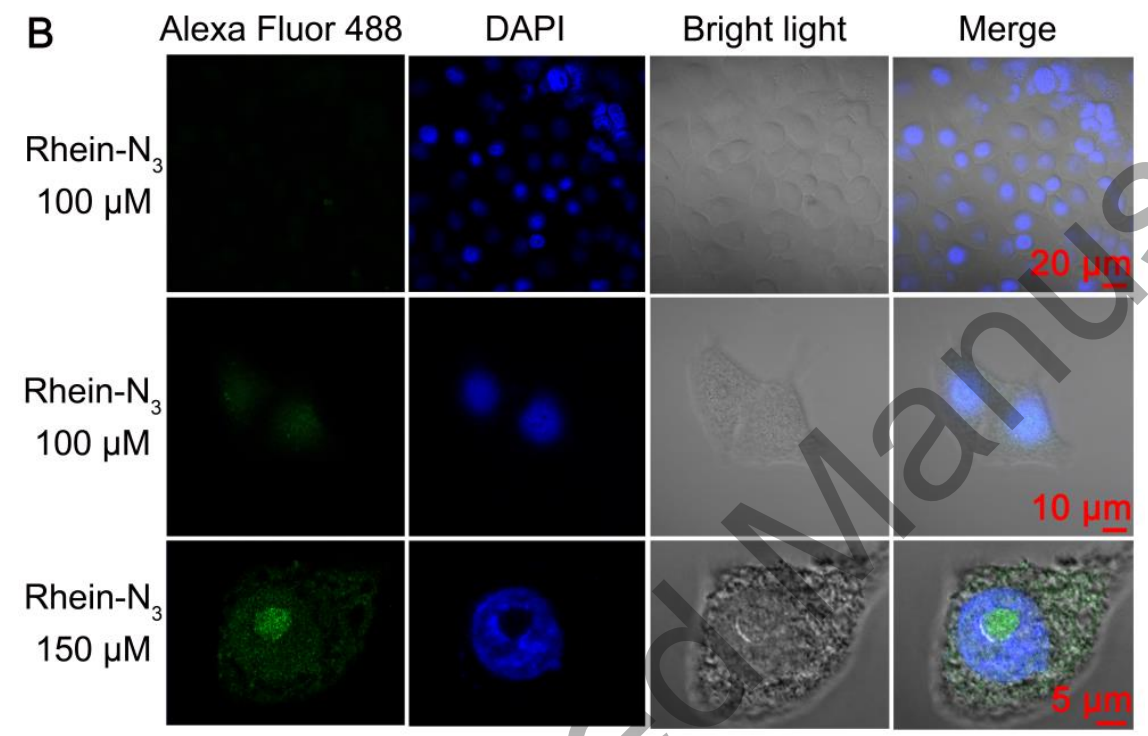

Figure 5. (A) Chemical structures of clickable agents and reaction scheme for bioorthogonal fluorescent labeling of rhein-azide derivative. (B) Confocal imaging of fixed live (upper panels) and necrotic (middle and bottom panels) A549 cells after being co-stained with Alexa Fluor 488 (green) and DAPI (blue). Live A549 cells were treated with $100 \mu \mathrm{M}$ rhein- $\mathrm{N}_{3}$ for $3 \mathrm{~h}$ and then were reacted with Alexa Fluor 488 alkyne in CuAAC reaction buffer. Necrotic A549 cells were treated with 100 or 150 $\mu \mathrm{M}$ rhein- $\mathrm{N}_{3}$ for $3 \mathrm{~h}$ and subsequently were reacted with Alexa Fluor 488 alkyne in CuAAC reaction buffer. (Reproduced from reference [76] with permission from the Centre National de la Recherche Scientifique (CNRS) and the Royal Society of Chemistry). 